\title{
FINE STRUCTURE OF BRIGHTNESS, VELOCITY AND MAGNETIC FIELD IN THE PENUMBRA
}

\author{
W. Mattig and J.P. Mehltretter \\ (Fraunhofer-Institut, Freiburg i.Br., Germany)
}

As a preparation for the observational program of the first flight of the 'SpectroStratoscope' balloon-borne solar telescope, an attempt was made with the $35-\mathrm{cm}$ aperture domeless Coudé refractor of the Capri Station of Fraunhofer Institute to obtain spectra of penumbral fine structure.

A polarizing image-splitting device (quarter-wave plate and Wollaston prism in front of the spectrograph slit) was used in order to obtain simultaneous spectra of the two states of circular polarization, containing the magnetically sensitive line $\lambda$ 6302.499 of FeI (Landé factor 5/2). This line has two sharp telluric lines $(\lambda 6302.000$ and $\lambda 6302.764$ of $\mathrm{O}_{2}$ ) as immediate neighbors serving as wavelength reference.

The exposure time of the double spectra was $15 \mathrm{sec}$ on Kodak 103a-E film. The image scale on the slit is 5.9 per $\mathrm{mm}$, and the reciprocal dispersion $6.92 \mathrm{~mm} / \AA$.

A series of spectra was taken under very good seeing conditions, May 29, 1967 of the spot $17^{\circ} \mathrm{S}, 44^{\circ} \mathrm{W}$, classification $\mathrm{J} 1$. The slit was set onto the penumbra at the limb's distant side, nearly tangential (deviation $20^{\circ}$ ) to the solar limb. The exposures were triggered by constantly watching the signal of the seeing monitor and thus selecting the periods of best seeing. Nevertheless, the individual spectra of this series have different resolution. So far, only the best spectrum, shown in Figure 1, has been analyzed.

Measurements of the spatial variation (along the slit height) were made of:

(a) the intensity $I(x)$ of the local continuum,

(b) the radial velocity $v(x)$,

(c) the component $H^{\prime}(x)=H(x) \cos \gamma(x)$ of the magnetic field,

(d) the line asymmetry $a(x)$,

by the following two methods:

Firstly, from a total of 200 microdensitometer tracings covering the two spectra in steps 0.45 apart, the intensity of the local continuum, the wavelength of the line center, and the line asymmetry have been determined.

Secondly, an equidensitogram (Figure 2) has been made by photographic procedures (Sabattier effect), and the line position and line width have been measured in steps 0.35 apart. In this case, the apparent width of the telluric lines was taken as an equivalent to the intensity of the local continuum. 


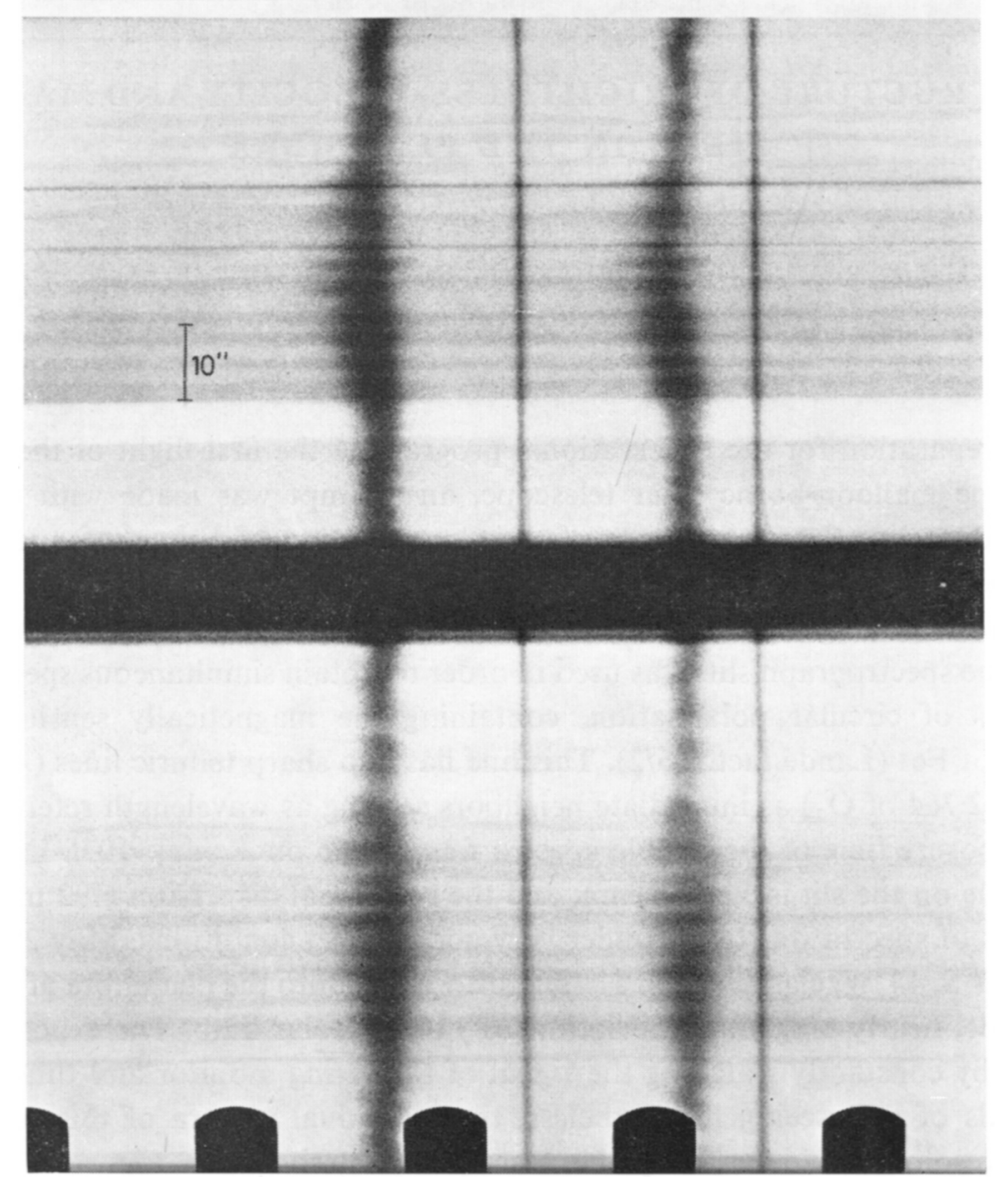

Fig. 1. Spectrogram showing penumbral fine structure, May 29, 1967, Spot $17^{\circ} \mathrm{S}, 44^{\circ} \mathrm{W}$.

The exact superposition of the two spectra and the measured values (wavelength) was accomplished by fitting the intensity curves (or line-width curves of the telluric lines) together.

Figure $3 \mathrm{a}$ summarizes the results obtained from the microdensitometer tracings, Figure $3 b$ those from the equidensitogram.

Although in Figure $3 a$ the velocity and intensity fluctuations are not well pronounced over the whole section of the penumbra, a good correlation appears between bright elements, smaller than average velocity, and stronger than average magnetic field.

In Figure $3 b$, the fluctuation of the magnetic field is small, but fluctuations of velocity and 'intensity' show the same correlation as in Figure 3a. 

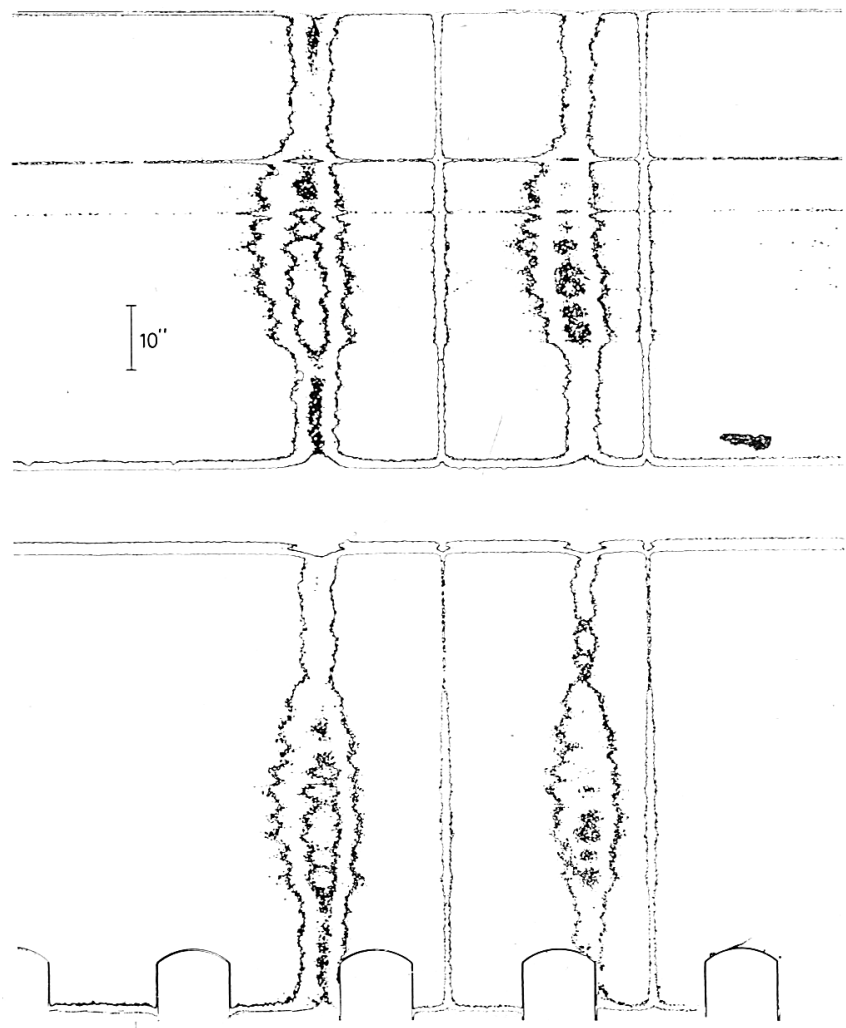

Fig. 2. Equidensitogram of the spectrum shown in Figure 1.

We would conclude, therefore, that there is a real correlation in the penumbra of

(1) bright elements, strong magnetic-field component, small radial velocity,

(2) dark elements, weak magnetic-field component, high radial velocity.

Further results can be summarized as follows:

The mean penumbral intensity is 0.75 of the photospheric intensity. The measured peak-to-peak fluctuation of intensity is about $10 \%$.

The average radial velocity observed is $1 \mathrm{~km} / \mathrm{sec}$. The peak-to-peak fluctuation is different depending on the height in the line contour. At the line center, $\Delta v$ (Max.Min.) is $700-800 \mathrm{~m} / \mathrm{sec}$, somewhat higher in the contour (where the line width is about $200 \mathrm{~m} \AA$ ), we find $\Delta v \approx 500 \mathrm{~m} / \mathrm{sec}$, hence $\Delta v / v=0 \cdot 2$ to $0 \cdot 4$, depending on the line contour.

The average magnetic-field component $H^{\prime}$ is about 700 gauss, the fluctuation $\Delta H^{\prime}$ about 200-300 gauss, hence $\Delta H^{\prime} / H^{\prime}=0 \cdot 3$.

Clearly, since the component $H(x) \cos \gamma(x)$ is observed, any fluctuation may be due to either $H(x)$ itself or due to variation of the inclination angle $\gamma(x)$. Moreover, 

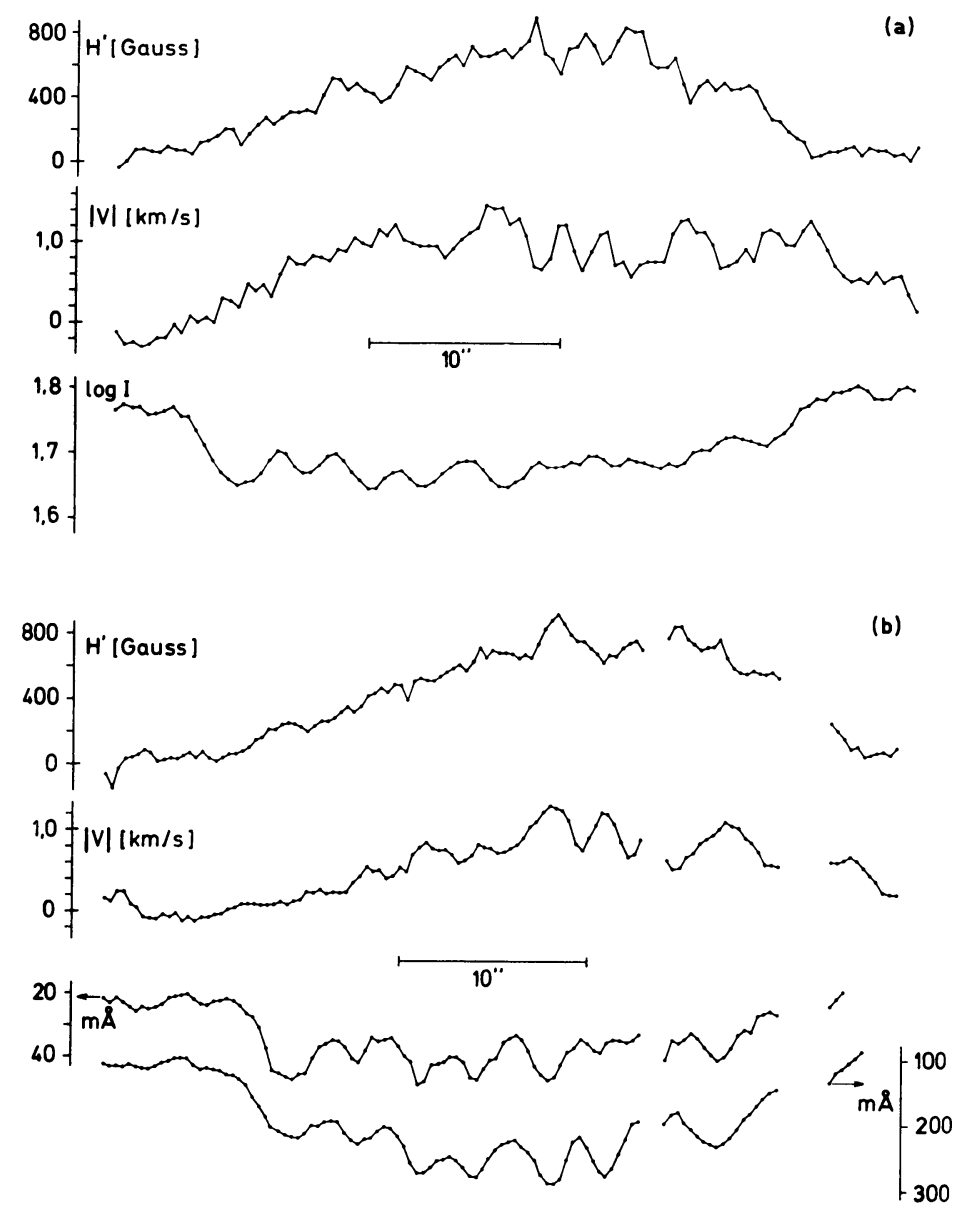

FIG. 3. (a) Results of analysis of microdensitometer tracings. (b) Results of analysis obtained from the equidensitograms.

since the line is far from being completely split, the values given may be lower than the actual $H^{\prime}$.

The asymmetry of the iron line was found to be essentially the same in both spectra, so we are allowed to conclude that the asymmetry is produced by unresolved velocity fluctuations rather than magnetic-field fluctuations. The same follows from the contour dependence of the measured $v$, and from the fact that the fluctuations in line width measured on the equidensitogram are approximately the same for both spectra.

In discussing the observation reported above, it should be stated that the spatial resolution ( 1 " to $\left.2^{\prime \prime}\right)$ of the spectrum is far from being sufficient for detecting individuat penumbral filaments (diameter $<0.4$, distance $2^{\prime \prime}$ or less). Most likely, the brighl elements in the spectrum represent accumulations of penumbral filaments. This fact 
is supported by the behaviour of the center-line of the line contour, which occasionally shows distinct steps.

As far as the correlation between brightness and velocity is concerned, the results are in agreement with earlier findings of Beckers (1966), and in contradiction with the hypothesis put forward by Schröter (1965). Clearly, more and even better observations are needed in order to make a sound study of the phenomena possible.

\section{References}

Beckers, J.M. (1966) in Convegno sulle macchie solari, Florence, p. 186.

Schröter, E.H. (1965) Z. Astrophys., 62, 228, 256.

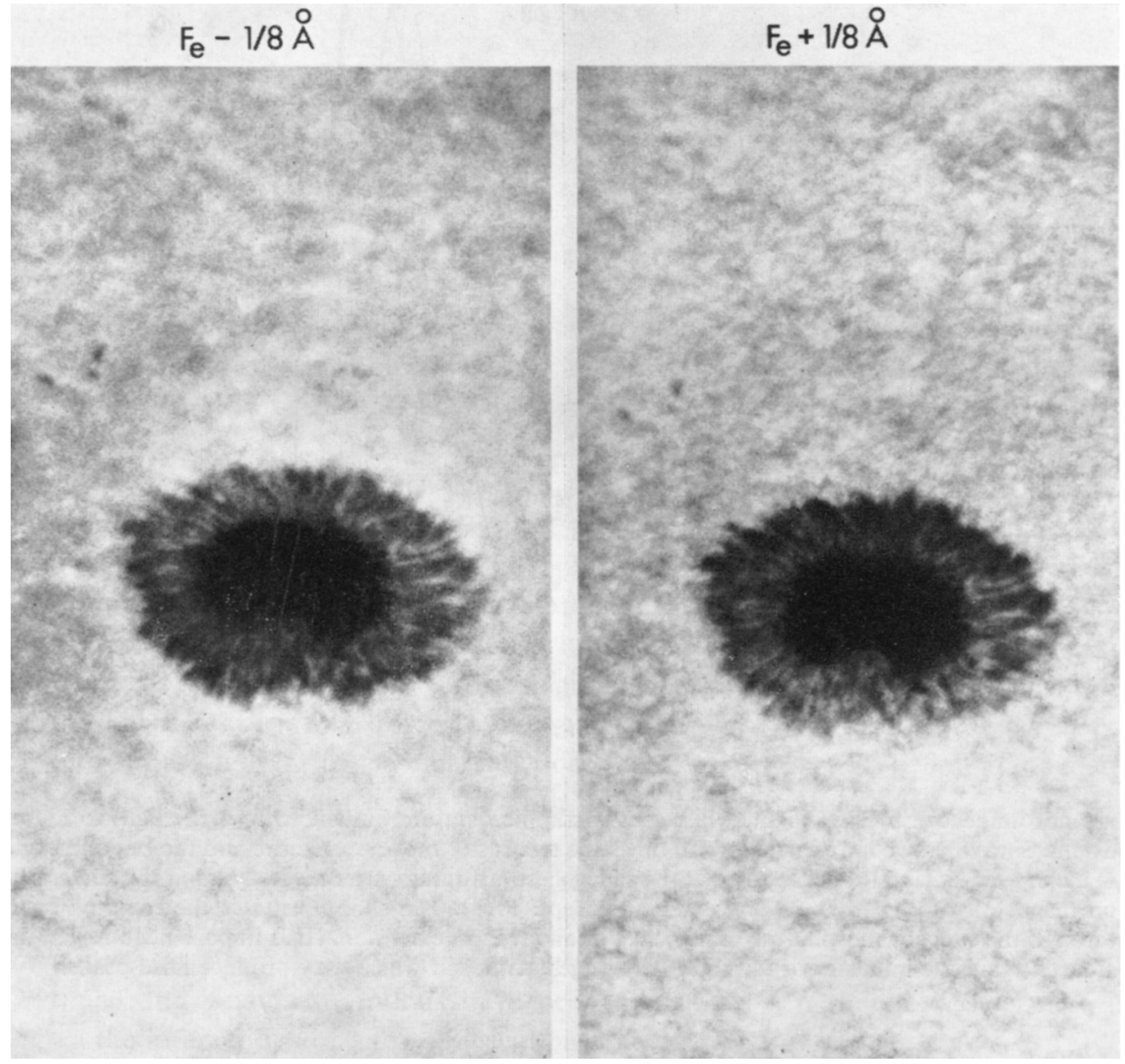

FIG. 4. Sunspot filtergrams $\lambda 6569.2+1 / 8$ A at radius vector 0.7 . Limb direction is vertically upward (Sacramento Peak Observatory). 


\section{DISCUSSION}

Beckers: From the preliminary results reported in an earlier paper Schröter and myself found the dark penumbral regions to show a magnetic-field enhancement. This disagrees with your measurements. We want to investigate this further, however, before being definite. [See Figure 4.]

Observations which I made of the Evershed velocities with a Zeiss $1 / 4 \AA$ filter shows the Evershed velocities to be located in the dark penumbral regions. This agrees with Dr. Mattig's measurements.

Severny: It is a pleasure to realize that the fine structures inside sunspots we found in 1959 (Astr. Zu $36,208)$ are now confirmed in the work of Dr. Beckers and Dr. Mattig. That time we found that the magnetic field even inside the umbra shows small peaks (or points, as calls them Dr. Beckers) about $30 \%$ of mean value and concentrated in regions comparable with the resolution of our magnetographic records $(\sim 2 " .5)$. [See Figure 5.] Magnetographic records have the advantage that they

\subsection{6}

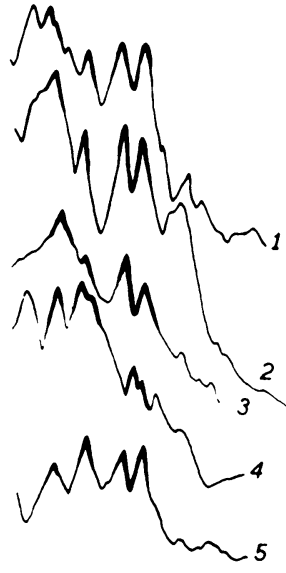

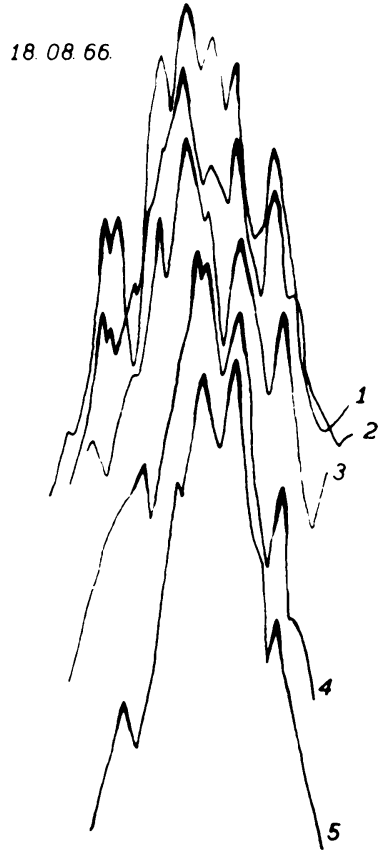

FIG 5.

are not influenced by stray light, while spectrographic data are influenced by stray light to higher extent. Since then we have made several times the records of the fine structure and the last ones were made in 1965-66 and they are shown at the slide (these last results are delivered also at the conference on magnetohydrodynamics in Sopot, Poland, Sept. 1966). The slide illustrates the repeated scans through the sunspot showing the same peaks of about $2^{\prime \prime}$ in extension. (It is important to repeat the scan to avoid the influence of trembling of images at the slit which may produce false peaks.) 